Article

\title{
Reconstruction of Mimetic Gravity in a Non-Singular Bouncing Universe from Quantum Gravity
}

\author{
Marco de Cesare \\ Department of Mathematics and Statistics, University of New Brunswick, Fredericton, NB E3B 5A3, Canada; \\ marco.de_cesare@unb.ca
}

Received: 1 April 2019; Accepted: 4 May 2019; Published: 7 May 2019

\begin{abstract}
We illustrate a general reconstruction procedure for mimetic gravity. Focusing on a bouncing cosmological background, we derive general properties that must be satisfied by the function $f(\square \phi)$ implementing the limiting curvature hypothesis. We show how relevant physical information can be extracted from power-law expansions of $f$ in different regimes, corresponding e.g., to the very early universe or to late times. Our results are then applied to two specific models reproducing the cosmological background dynamics obtained in group field theory and in loop quantum cosmology, and we discuss the possibility of using this framework as providing an effective field theory description of quantum gravity. We study the evolution of anisotropies near the bounce, and discuss instabilities of scalar perturbations. Furthermore, we provide two equivalent formulations of mimetic gravity: one in terms of an effective fluid with exotic properties, the other featuring two distinct time-varying gravitational "constants" in the cosmological equations.
\end{abstract}

Keywords: mimetic gravity; limiting curvature; bouncing cosmology; quantum gravity; effective field theory

\section{Introduction}

The resolution of spacetime singularities is one of the main expected consequences of quantum gravity. In cosmology, the realization of such a possibility would lead to the replacement of the Big Bang singularity by a smooth spacetime region, e.g., a bounce, with profound implications for our understanding of the earliest stages of cosmic expansion and of the initial conditions for our universe. Non-singular bouncing cosmologies have been extensively studied and may represent an alternative to the inflationary scenario [1] with specific observational signatures (see also [2]). Resolution of the initial singularity in cosmology has been achieved in various approaches based on a loop quantization of the gravitational field, such as loop quantum cosmology (LQC) [3,4], group field theory (GFT) condensate cosmology [5,6], and quantum reduced loop gravity [7]; more specifically, both in LQC and in GFT the initial singularity is replaced by a regular bounce, marking the transition from a contracting phase to an expanding one.

One of the main open problems that is common to all background-independent approaches to quantum gravity is the derivation of an effective field theory taking into account effects due to the underlying discreteness of spacetime at the Planck scale. In fact, at present very little is known about quantum gravity beyond perfect homogeneity, although efforts to include inhomogeneities in the description of an emergent universe from full quantum gravity are underway [8-10]. One possible alternative approach then consists of considering modifications of general relativity that can reproduce known features of a given quantum gravity theory. The hope is that by doing so, we can gain insight (at least qualitatively) into the consequences of quantum gravitational effects in different regimes. In this work, we adopt the framework of limiting curvature mimetic gravity and examine in detail the problem of reconstructing the theory from the evolution of the cosmological background, with particular 
attention to the case of a bouncing background. Such a theory should then be regarded as a toy model for an effective description of quantum gravity $[11,12]$ and can be used to study its phenomenological consequences. Possible applications include e.g., the dynamics of inhomogeneous and anisotropic degrees of freedom in cosmology, and black holes.

The idea of limiting curvature as a possible solution to the singularities of general relativity was first envisaged in Ref. [13-16], and subsequently implemented in modifications of the Einstein-Hilbert action including higher-order curvature invariants in Refs. [17-20]. An alternative proposal for constructing a gravitational theory with a built-in limiting curvature scale was put forward in Ref. [21] as an extension of mimetic gravity. This is achieved by including in the action functional a (multivalued) potential term $f$ depending on the d'Alembertian of a scalar field $\phi$. Upon closer inspection, such a potential turns out to depend on the expansion scalar $\chi$ of a privileged irrotational congruence of time-like geodesics, singled out by the so-called mimetic constraint [22]. On a cosmological spacetime, $f(\chi)$ reduces to a function of the Hubble rate [23]. Multivaluedness of the potential is necessary for a consistent realization of bouncing cosmologies in this framework [22,24-26]. Non-singular black hole solutions have been studied in Refs. [27,28].

The particular model proposed in Ref. [21] exactly reproduces the effective dynamics obtained in (flat, isotropic) homogeneous LQC. Thus, all curvature invariants are bounded throughout spacetime by a limiting curvature scale, which is in turn related to the existence of a critical value for the energy density of matter at the bounce. From the point of view of quantum gravity, it is natural to require that the limiting curvature scale be Planckian. In Ref. [12] a broader class of theories was identified in the degenerate higher-order scalar tensor theories (DHOST) family, all reproducing the effective dynamics of LQC; these models can be further extended by the inclusion of a term corresponding to the spatial curvature. The relation between the model of Ref. [21] and effective LQC was further investigated in Refs. [11,29] from a Hamiltonian perspective, showing that the equivalence holds in the spatially flat, homogeneous, and isotropic sector; however, the correspondence is lost in the anisotropic case. Nevertheless, even for anisotropic cosmologies the solutions of the two models are qualitatively similar [11,29]. The mimetic model of Ref. [21] has been recently generalized in Ref. [22], where a limiting curvature mimetic gravity theory was reconstructed so as to exactly reproduce the background evolution obtained from GFT condensates in Ref. $[5,6]$; the effective dynamics of homogeneous LQC is then recovered as a particular case for some specific choice of the parameters of the model.

This paper has two main goals. The first one is to give a general account of theory reconstruction in mimetic gravity, showing how essential information about background evolution (e.g., the critical energy density, the bounce duration, and the equation of state of effective fluids) is encoded in the function $f(\chi)$, particularly in its asymptotic behavior in regimes of physical interest. The case of a generic bouncing background is examined in detail, although our methods have a much broader applicability. We provide general prescriptions for the matching of the different branches of the multivalued function $f(\chi)$, which are necessary in order to obtain a smooth evolution of the universe, thus generalizing the analysis of matching conditions in Ref. [22]. Our second goal is to study in detail the properties of mimetic gravity theories with the same background evolution as obtained in non-perturbative approaches to quantum gravity. Specifically, we analyze the model of Ref. [22] reproducing the background evolution obtained from GFT condensates, and compare it to the special case corresponding to the LQC effective dynamics. We study the evolution of anisotropies near the bounce in a Bianchi I spacetime, including the effects of hydrodynamic matter with generic equation of state, thus extending the results of Ref. [21]. As in the model of Ref. [21], our more general results also show that the smooth bounce is not spoiled by anisotropies, which stay bounded during the bounce era. Instabilities in the inhomogeneous sector are also discussed. Moreover, given its relevance and simplicity, the particular case corresponding to the effective dynamics of LQC is analyzed separately.

The plan of the paper is as follows. The formulation of mimetic gravity is briefly reviewed in Section 2 . In Section 3 we discuss the reconstruction procedure. In Section 4 we focus on the model of Ref. [22]: 
we discuss the background evolution, exhibit the form of the function $f(\chi)$ and derive its expansion in its two branches, corresponding to the region around the bounce and to a large universe. The model of Ref. [22], which can be obtained as a particular case from our more general model, is discussed separately due to its relevance and simplicity. Section 5 is devoted to the study of anisotropies in a bouncing background. In Section 6 we provide an alternative description of the cosmological dynamics of mimetic gravity in terms of two effective gravitational "constants", both depending on the expansion rate of the universe. In Section 7 we discuss instabilities of scalar perturbations. We conclude with a discussion of our results in Section 8.

We choose units such that $8 \pi G=1$. Landau-Lifshitz conventions for the metric signature $(+---)$ are adopted.

\section{Mimetic Gravity and Its Cosmology}

The version of mimetic gravity considered in Ref. [21] is based on the action

$$
S\left[g_{\mu v}, \phi, \lambda, \psi\right]=\int \mathrm{d}^{4} x \sqrt{-g}\left(-\frac{1}{2} R+\lambda\left(g^{\mu v} \partial_{\mu} \phi \partial_{\nu} \phi-1\right)+f(\chi)+L_{\mathrm{m}}\left(\psi, g_{\mu v}\right)\right),
$$

with $\chi=\square \phi$. The gravitational sector consists of the metric $g_{\mu v}$ and the scalar field $\phi$. The Lagrange multiplier $\lambda$ enforces the mimetic constraint

$$
g^{\mu v} \partial_{\mu} \phi \partial_{\nu} \phi=1
$$

We have included a matter Lagrangian $L_{\mathrm{m}}$, where $\psi$ represents a generic matter field, coupled to $g_{\mu \nu}$ only and not to $\phi$. Due to the term $f(\chi)$, the action (1) represents a higher-derivative extension of the original mimetic gravity theory of Ref. [30]. ${ }^{1}$

Due to the mimetic constraint, the vector field $u^{\mu}=g^{\mu v} \partial_{\nu} \phi$ has unit norm and generates an irrotational congruence of time-like geodesics (see Ref. [22] for more details). Thus, the theory admits a preferred foliation ${ }^{2}$ with time function $t=\phi$ and time-flow vector field $u^{\mu} \frac{\partial}{\partial x^{\mu}}=\frac{\partial}{\partial t}$. The quantity $\chi$, defined above, can be expressed as $\chi=\nabla^{\mu} u_{\mu}$ and represents the expansion of the geodesic congruence generated by $u^{\mu}$. In FLRW spacetime, one has $\chi=3 H$, where $H$ denotes the Hubble rate. It is for this reason that the term $f(\chi)$ in the action (1) plays an important role in the cosmological applications of the model, since for a homogenous and isotropic background $f(\chi)$ reduces to a function of the Hubble rate only. This is a crucial property of the model, which allows for a straightforward theory reconstruction procedure, starting from a given cosmological background evolution. This aspect will be analyzed in detail in Section 3.

It is worth stressing that although the action for mimetic gravity includes higher-derivative terms through $f(\chi)$, the equations of motion are second order. In fact, mimetic gravity is a particular case of so-called DHOST, which are characterized by the absence of Ostrogradski ghost [23,35]. Nevertheless, compared to general relativity, the mimetic gravity theory described by (1) has an extra propagating scalar degree of freedom if $f_{\chi \chi} \neq 0[33,36]$. Importantly, this is always a source of instabilities in the theory, as discussed in Section 7.

The field equations read as [21]

$$
G_{\mu v}=T_{\mu v}^{\psi}+\tilde{T}_{\mu v}
$$

where the matter stress-energy tensor is defined as usual

1 The original formulation of mimetic gravity of Ref. [30] relied on a singular disformal transformation [31] (see also Ref. [23]). An equivalent formulation with a Lagrange multiplier implementing the constraint (2) was given in Ref. [32]. The latter represents the starting point for further generalizations of the model considered in Refs. [21,33]. See also the review [34].

2 Such a gauge choice corresponds to unit lapse and vanishing shift, i.e., $N=1$ and $N^{i}=0$. 


$$
T_{\mu v}^{\psi}=\frac{2}{\sqrt{-g}} \frac{\delta S_{\mathrm{m}}}{\delta g^{\mu v}}
$$

and the extra term in Equation (3) is an effective stress-energy tensor arising from the $\phi$-sector of the action (1)

$$
\tilde{T}_{\mu \nu}=2 \lambda \partial_{\mu} \phi \partial_{\nu} \phi+g_{\mu \nu}\left(\chi f_{\chi}-f+g^{\rho \sigma} \partial_{\rho} f_{\chi} \partial_{\sigma} \phi\right)-\left(\partial_{\mu} f_{\chi} \partial_{\nu} \phi+\partial_{\nu} f_{\chi} \partial_{\mu} \phi\right) .
$$

The Lagrange multiplier $\lambda$ can be eliminated by solving the following equation

$$
\square f_{\chi}-2 \nabla^{\mu}\left(\lambda \partial_{\mu} \phi\right)=0,
$$

which can be obtained by varying the action with respect to $\phi$. Equation (6) can be interpreted as a conservation law for the Noether current associated with the global shift-symmetry of the action (1), see Refs. [22,37]

Considering a flat FLRW model $\mathrm{d} s^{2}=\mathrm{d} t^{2}-a^{2}(t) \delta_{i j} \mathrm{~d} x^{i} \mathrm{~d} x^{j}$, the field Equation (3) lead to a modification of the Friedmann and Raychaudhuri equations

$$
\begin{aligned}
& \frac{1}{3} \chi^{2}=\rho+\tilde{\rho}+M \\
& \dot{\chi}=-\frac{3}{2}[(\rho+P)+(\tilde{\rho}+\tilde{P})+M] .
\end{aligned}
$$

The quantities introduced in Equations (7) and (8) are defined as follows: $\rho$ and $P$ denote the energy density and pressure of ordinary matter, whereas $\tilde{\rho}$ and $\tilde{P}$ represent the corresponding quantities for the effective fluid, given by

$$
\begin{aligned}
& \tilde{\rho}=\chi f_{\chi}-f, \\
& \tilde{P}=-\left(\tilde{\rho}+f_{\chi \chi} \dot{\chi}\right) .
\end{aligned}
$$

The properties of the effective fluid for a quadratic $f(\chi)$ were studied in Ref. [37]. Lastly, we have $M=\frac{C}{a^{3}}$, where $C$ is an integration constant for Equation (6). The quantity $M$ represents the energy density of so-called mimetic dark matter [30]. We note that for vanishing $f$ the action (1) describes irrotational dust minimally coupled to gravity, corresponding to a particular case of the Brown-Kuchař action $[38] .^{3}$ Finally, we observe that the effective fluid satisfies the continuity equation

$$
\dot{\tilde{\rho}}+\chi(\tilde{\rho}+\tilde{P})=0 .
$$

\section{Theory Reconstruction}

We henceforth consider a spatially flat, homogeneous, and isotropic universe, as described by the FLRW line element $\mathrm{d} s^{2}=\mathrm{d} t^{2}-a^{2}(t) \delta_{i j} \mathrm{~d} x^{i} \mathrm{~d} x^{j}$. The proper time gauge $N=1$ will be used throughout. The spacetime geometry is then fully characterized by the evolution of a single degree of freedom: the scale factor $a(t)$. Given a theory of gravity with second order field equations, cosmological solutions can be represented as trajectories in the plane $(a, \chi)$. In general relativity, the trajectories are determined by the Friedmann equation

$$
\frac{1}{3} \chi^{2}=\sum_{i} \rho_{i}
$$

Here the quantities $\rho_{i}$ denote the energy density of different matter species. For the sake of simplicity, we can assume that all matter species are non-interacting and have constant equation of state parameters $w_{i}$. Thus, we have $\rho_{i}=c_{i} V^{-\left(w_{i}+1\right)}$, where $c_{i}$ are constants depending on the initial

3 See also Refs. [39,40]. 
conditions and $V=a^{3}$ is the proper volume of a unit comoving cell. It is convenient to introduce a new variable $\eta=V^{-1}$, so that the Friedmann equation can be re-expressed as

$$
\frac{1}{3} \chi^{2}=\sum_{i} c_{i} \eta^{w_{i}+1}
$$

Such a parametrization is particularly useful in bouncing cosmologies, where $\eta$ has a bounded range. In the following, we will denote by $\Gamma$ the trajectory in the $(\eta, \chi)$ plane given by Equation (13).

Despite the derivation given above, based on the standard Friedmann equation, Equation (13) has a broader applicability. In fact, it also holds in a more general class of modified gravity theories and quantum cosmological models, provided that the corrections to the standard Friedmann equation can be described - at an effective level—as perfect fluids. Such effective fluids may have exotic properties and, depending on the model, can violate the energy conditions. This is the case, for instance, in the effective dynamics of both LQC and GFT condensate cosmology. In fact, having a bounce requires that both the weak and the null energy conditions must be violated due to the effective fluids. The former violation is necessary to accommodate for a vanishing expansion, see Equation (13). The latter violation follows instead from the requirement that $\dot{\chi}>0$ at the bounce, and from the Raychaudhuri equation including effective fluids contributions

$$
\dot{\chi}=-\frac{3}{2} \sum_{i}\left(\rho_{i}+P_{i}\right) .
$$

It is important to observe that in general Equation (13) allows us to define $\chi$ as a function of $\eta$ only locally. In fact, in bouncing models, the function $\chi(\eta)$ has (at least) two branches. More branches are possible if one allows, e.g., for intermediate recollapse eras; we shall disregard this possibility in the following for simplicity. For a universe undergoing a single bounce, the trajectory $\Gamma$ has the profile depicted in Figure 1 . The bounce is represented by the point $B=\left(\eta_{\max }, 0\right)$, where $\Gamma$ and the $\eta$ axis intersect orthogonally. Since we are assuming a flat spatial geometry, both endpoints of $\Gamma$ will have $\eta=0$ if the weak energy condition is satisfied for a large universe. The value of $\chi$ at the endpoints is determined by the equation of state of the dominant matter species in such a regime: for $w>-1$ one has that $\chi$ vanishes as $\eta$ tends to zero, for $w=-1$ (cosmological constant) $\chi$ approaches a constant value. We note that for $w+1>0$ the two endpoints coincide with the origin; moreover, for $-1<w<1$ the trajectory $\Gamma$ intersects the $\eta$ axis orthogonally at the origin, whereas for $w \geq 1$ it has a cusp.
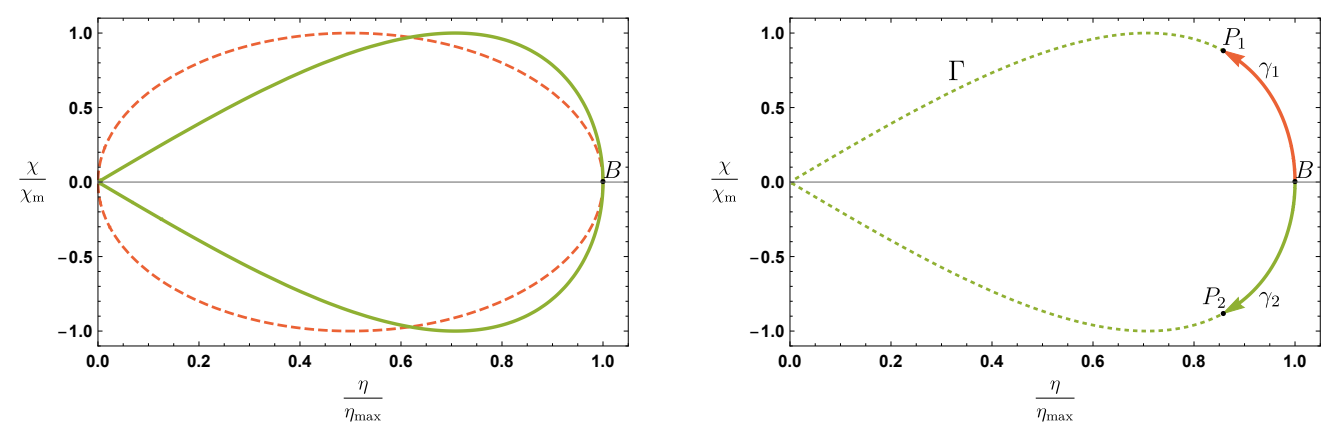

Figure 1. Trajectories $\Gamma$ in the $(\eta, \chi)$ plane for a (symmetric) bouncing universe. The upper half-plane corresponds to the expanding phase, whereas the lower half-plane describes the contracting phase. The bounce is represented by the point $B$, where the expansion rate vanishes, and the scale factor attains its minimum (correspondingly $\eta$ is maximized). The left figure shows the trajectory $\Gamma$ for a universe filled with a scalar field (thick green line), or dust (dashed orange line); parameters are chosen so that the two trajectories are characterized by the same critical density $\rho_{c}$ and limiting expansion rate $\chi_{\mathrm{m}}$. The right figure shows the two integration contours $\gamma_{1}, \gamma_{2}$ used in Equation (17). 


\subsection{Reconstruction Procedure}

Given a background evolution as specified by the trajectory $\Gamma$, it is possible to apply a reconstruction procedure that allows us to uniquely determine the function $f(\chi)$ in the mimetic gravity action (1). The method illustrated in this section extends to a generic background evolution the procedure applied in Refs. $[22,25,26]$ and ensures that appropriate matching conditions are implemented at the branching points. ${ }^{4}$ We start by rewriting Equation (7), using Equation (9), as

$$
\frac{\chi^{2}}{3}\left[1-3 \frac{\mathrm{d}}{\mathrm{d} \chi}\left(\frac{f}{\chi}\right)\right]=\rho .
$$

The solution to this equation can be obtained by quadrature, and is given by

$$
f(\chi)=\frac{\chi}{3} \int_{A}^{P} \mathrm{~d} \chi\left(1-\frac{3 \rho(\eta(\chi))}{\chi^{2}}\right)+\bar{c} \chi
$$

where $\bar{c}$ is an integration constant. The integral is computed along an arc of curve $\gamma \subseteq \Gamma$ with endpoints $A$ and $P$, representing a fixed reference point and a generic point on $\Gamma$, respectively.

In bouncing cosmologies, the background dynamics is characterized by the existence of a limiting curvature scale, which is attained at the bounce. In turn, this scale is related to the existence of a maximum expansion rate, which will be denoted by $\chi_{\mathrm{m}} \equiv \max _{\Gamma} \chi$, see Figure 1 . In this class of models, it is convenient to take the bounce as a reference point, i.e., $A \equiv B$ in Equation (16). Since the energy density of matter is given as a function of the inverse volume, i.e., $\rho=\rho(\eta)$, the explicit computation of the integral (16) requires the determination of the inverse function $\eta(\chi)$. In general, such an inverse function exists only locally. This implies that in bouncing models the function $f(\chi)$ given by Equation (16) must be multivalued as a function of $\chi .^{5}$ More precisely, in models with a single bounce $f(\chi)$ has two branching points where $\chi$ attains its extrema, one in the expanding phase, the other in the contracting phase. For a generic bouncing background $f(\chi)$ would have three branches, each corresponding to one of the three branches of the inverse functions $\eta(\chi)$. Thus, one branch $f_{\mathrm{B}}$ corresponds to the bounce phase, and two (a priori distinct) branches $f_{\mathrm{L}}^{c}, f_{\mathrm{L}}^{e}$ correspond to the regions away from the bounce in the contracting and expanding phase, respectively. We will refer to the latter as the large universe branches, characterized by $\dot{\chi}<0$. As shown in Section 3.2, for symmetric bounces the two branches $f_{\mathrm{L}}^{c}, f_{\mathrm{L}}^{e}$ can be identified, provided that an appropriate choice is made for the integration constant in Equation (16).

We remark that our solution for $f$ is continuous on $\Gamma$ by construction. The derivative $f_{\chi}$ is also continuous, except at the origin $\chi=\eta=0 .{ }^{6}$ This ensures that the energy density of the effective fluid, Equation (9), is continuous throughout cosmic history. Thus, the matching conditions prescribed in Ref. [22] are automatically implemented in Equation (16). As a general property of this class of models $f_{\chi \chi}$ diverges at the branching points, see discussion in Section 4.

After computing the integral in Equation (16), the reconstructed action for mimetic gravity can then be obtained by replacing $\chi \rightarrow \square \phi$ in the result. Clearly, the value of the integration constant $\bar{c}$ has no influence on the equations of motion, since the linear term contributes a total divergence to the action (1).

4 We note that a different version of mimetic gravity is considered in Ref. [26] that agrees at the background level with the one presently considered. However, the two theories will differ in general at the level of perturbations.

5 We observe that for models entailing a single bounce, the solution (16) is single-valued if regarded as a function of the pair $(\chi, \dot{\chi})$.

6 The fact that the origin is a singular point in the parametrization adopted here should not be too surprising: in fact, it corresponds to the infinite volume limit of both contracting and expanding branches. In a flat universe these are clearly two disconnected regimes. 


\subsection{Bounce Asymptotics}

For a symmetric bounce model, the function $f(\chi)$ is even, provided that an appropriate choice of the integration constant is made in Equation (16). In fact, defining $P_{1}=(\eta, \chi)$ and $P_{2}=(\eta,-\chi)$, with $\eta$ and $\chi$ satisfying the background equation, one has

$$
\int_{\gamma_{1}}^{P_{1}} \mathrm{~d} \chi\left(1-\frac{3 \rho(\eta(\chi))}{\chi^{2}}\right)=-\int_{\gamma_{2}}^{P_{2}} \mathrm{~d} \chi\left(1-\frac{3 \rho(\eta(\chi))}{\chi^{2}}\right) .
$$

Thus, the integral is odd. The curves $\gamma_{1}$ and $\gamma_{2}$ are depicted in Figure 1. Using Equations (17) and (16), it is then straightforward to show that setting $\bar{c}=0$ leads to $f(\chi)=f(-\chi)$. In the following, we shall restrict our attention to symmetric bounce models and assume that $f(\chi)$ be even, unless otherwise stated.

The value of the function $f$ at the bounce is independent from all other details of cosmic history. It can be computed as a limit of Equation (16). Denoting by $f_{\mathrm{B}}$ the bounce branch of the multivalued function $f$, we have

$$
f_{\mathrm{B}}(0)=\lim _{P \rightarrow B} \frac{\chi}{3} \int_{\underset{\gamma}{P}}^{P} \mathrm{~d} \chi\left(1-\frac{3 \rho(\eta(\chi))}{\chi^{2}}\right)=\lim _{\chi \rightarrow 0} \frac{\chi}{3} \int_{0}^{\chi} \mathrm{d} \chi\left(1-\frac{3 \rho(\eta(\chi))}{\chi^{2}}\right)=\rho_{\mathrm{C}},
$$

where $\rho_{\mathrm{c}}$ is the critical density, i.e., the maximum of the energy density of matter, which is attained at the bounce. Since $f_{\mathrm{B}}$ is even by hypothesis, we have for $\chi \simeq 0$

$$
f_{\mathrm{B}}(\chi)=\rho_{\mathrm{c}}+\frac{1}{2 !} \vartheta \chi^{2}+\mathcal{O}\left(\chi^{4}\right)
$$

where we introduced the notation $\vartheta=\left.\left(f_{\mathrm{B}}\right)_{\chi \chi}\right|_{0}$. Hence, it follows that the energy density of the effective fluid at the bounce is given by $\tilde{\rho}=-\rho_{\mathrm{c}}$. The sign of the second derivative can be determined by the requirement that the effective fluid must also violate the null energy condition (NEC) at the bounce. In fact, using Equation (10) we have

$$
\tilde{\rho}+\tilde{P}=-\left(f_{\mathrm{B}}\right)_{\chi \chi} \dot{\chi}<0
$$

Since $\dot{\chi}>0$ at the bounce, we conclude $\vartheta>0$.

NEC violation also allows us to derive an upper bound for the duration of the bounce in limiting curvature mimetic gravity. To prove such a statement, let us assume that at the bounce the most relevant contributions to the energy density are due to the effective fluid and to a perfect fluid with equation of state parameter $w$. The condition $\dot{\chi}>0$, which must be valid in a neighborhood of the bounce, implies

$$
\rho+p+\tilde{\rho}+\tilde{P}<0
$$

In turn, Equation (21) implies

$$
(1+w) \rho_{c}-\left(f_{\mathrm{B}}\right)_{\chi \chi} \dot{\chi}<0
$$


For first-order bounce models ${ }^{7}$ during the bounce phase the expansion $\chi$ is well approximated by a linear function of time. We can estimate the time derivative of $\chi$ at the bounce as $\dot{\chi} \sim \frac{\chi_{\mathrm{m}}}{T}$, where $T$ is the bounce duration. Therefore, in this case we obtain from Equation (22)

$$
T \lesssim \frac{\vartheta \chi_{\mathrm{m}}}{\rho_{c}(1+w)}
$$

Typically, $\rho_{c} \sim \chi_{\mathrm{m}}^{2}$ and $\vartheta \sim \mathcal{O}(1)$, so that $T \lesssim \chi_{\mathrm{m}}^{-1}$. When such an approximation applies, the number of e-folds of expansion during the bounce phase is $N=\log \left(\frac{a(T)}{a_{\mathrm{B}}}\right) \lesssim \mathcal{O}(1)$. These considerations also apply to the models studied in Section 4 (see Equation (33) for the corresponding expansion of $f$ near the bounce). In fact, the estimate (23) agrees with the upper bound for the number of e-folds obtained in Ref. [42] for the so-called non-interacting model. We mention that the so-called fast-bounce models, considered e.g., in Ref. [43], are first-order bounces whose duration is much shorter than the time-scale linked to the maximum expansion rate, i.e., such that $T \ll \chi_{\mathrm{m}}^{-1}$; such a scenario can be realized in mimetic gravity by requiring $\left.\frac{\left(f_{\mathrm{B}}\right)_{\chi \chi}}{f_{\mathrm{B}}}\right|_{\chi=0} \ll \chi_{\mathrm{m}}^{-2}$.

\subsection{Late Time Asymptotics}

Considerations on the evolution of the universe at late times allow us to put restrictions on the leading order terms of the branch $f_{\mathrm{L}}$ around $\chi \simeq 0$. In fact, we observe that the effective fluid is characterized by a time-dependent equation of state parameter $\tilde{w}$, given by

$$
\tilde{w}=\frac{\tilde{P}}{\tilde{\rho}}=-\left(1+\frac{f_{\chi \chi}}{\tilde{\rho}} \dot{\chi}\right)
$$

where we used Equations (9) and (10). It is interesting to examine the case where the universe at late times is dominated by matter with equation of state $w$ and the effective fluid is sub-dominant, with $\tilde{w}$ approaching a constant value as $\chi \rightarrow 0$. Clearly, consistency of such assumptions requires $w<\tilde{w}$. The leading order term in the expansion of $f_{\mathrm{L}}(\chi)$ around $\chi \simeq 0$ is then given by

$$
f_{\mathrm{L}}(\chi) \simeq \lambda \chi^{2\left(\frac{\tilde{w}+1}{w+1}\right)}
$$

where $\lambda$ is a constant. In fact, since by hypothesis we must leading order $\chi \sim \eta^{\frac{1+w}{2}}$, Equation (25) implies $\tilde{\rho} \sim \eta^{1+\tilde{w}}$, consistently with our assumptions.

\section{Effective Approach to Quantum Gravitational Bouncing Cosmologies}

In Ref. [22] the reconstruction procedure outlined in Section 3 was successfully applied to the cosmological dynamics obtained from group field theory condensates in $[5,6]$. The evolution equation for such a model can be expressed in relational form by introducing a minimally coupled massless scalar field $\psi$ [44]. In fact, provided that its momentum be non-vanishing $p_{\psi} \neq 0, \psi$ is a monotonic function of $t$ and thus represents a perfect clock. For definiteness, we will assume $p_{\psi}>0$. Using the relational clock $\psi$ as time, the FLRW line element can be expressed as

$$
\mathrm{d} s^{2}=N^{2}(\psi) \mathrm{d} \psi^{2}-a^{2}(\psi) \delta_{i j} \mathrm{~d} x^{i} \mathrm{~d} x^{j},
$$

where the lapse function reads as

$$
N(\psi)=(\dot{\psi})^{-1}=p_{\psi}^{-1} a^{3}(\psi)
$$

\footnotetext{
7 The order of the bounce is defined as the positive integer $n$ such that $a^{(2 n)}\left(t_{\mathrm{B}}\right)>0$ is the lowest-order non-vanishing derivative of the scale factor at the bounce [41].
} 
We can define a relational Hubble rate as $\mathcal{H}=\frac{a^{\prime}}{a}$, where a prime denotes differentiation with respect to $\psi$. The expansion $\chi$ is related to $\mathcal{H}$ as follows

$$
\chi=3 p_{\psi} \frac{\mathcal{H}}{a^{3}} .
$$
$\left.V=a^{3}\right)$

The relational Friedmann equation governing the dynamics of GFT condensates reads as (recall

$$
\mathcal{H}^{2}=\frac{1}{6}+\frac{\alpha}{V}-\frac{\beta}{V^{2}},
$$

where $\alpha$ and $\beta>0$ are parameters depending on the details of the microscopic model, see Ref. $[5,6] .{ }^{8}$ An effective Friedmann equation with the same form as Equation (29) was obtained in the GFT models of Refs. [45,46]. The first term in Equation (29) is the contribution of the massless scalar field $\psi$, whereas the remaining two terms represent quantum gravitational corrections; in particular, the $\alpha$ term represents a correction to the effective dynamics of LQC. It must be stressed that for simplicity, we are neglecting interactions between GFT quanta, which would contribute additional terms to Equation (29). The cosmological consequences of interactions were considered in Ref. [42].

Changing time parametrization back to proper time and recalling $\eta=V^{-1}$, we have

$$
\frac{1}{3} \chi^{2}=p_{\psi}^{2}\left(\frac{1}{2} \eta^{2}+3 \alpha \eta^{3}-3 \beta \eta^{4}\right)
$$

The first term to the r.h.s. of Equation (30) gives the energy density $\rho_{\psi}$ of the scalar $\psi$; the quantum gravitational corrections (second and third terms) correspond instead to effective fluids with equation of state parameter $w=2,3$. The third term becomes important for large values of $\eta$ (i.e., small values of the scale factor); moreover, since $\beta>0$ such a term violates both the weak and the NECs, and is therefore responsible for the bounce. It must be noted that the bounce is symmetric for any choice of parameters in this model. The equation for $\dot{\chi}$ is

$$
\dot{\chi}=-\frac{3}{2} p_{\psi}^{2}\left(\eta^{2}+9 \alpha \eta^{3}-12 \beta \eta^{4}\right) .
$$

For further details on the effective fluid description of quantum gravity corrections in the effective Friedmann equation arising in the GFT approach, including interactions between quanta, the reader is referred to Refs. [42,47]. For a large universe (i.e., small $\eta$ ) the first term in Equation (30) becomes the dominant one: the standard Friedmann evolution is thus recovered, and the quantum gravity corrections are sub-leading.

The background evolution (30) can be exactly reproduced in mimetic gravity if the function $f(\chi)$ is given by [22]

$$
f(\chi)=\rho_{\psi}(\chi)+\frac{1}{3} \chi^{2}+\frac{p_{\psi}}{3 \sqrt{\beta}}|\chi|\left[\arctan \left(\frac{1}{\sqrt{\beta}} \frac{\mathrm{d}|\mathcal{H}|}{\mathrm{d} \eta}\right)+\frac{\pi}{2}\right] .
$$

By construction, the different branches of the multivalued function in Equation (32) satisfy matching conditions at the branching points, to ensure the regularity of cosmological evolution. Around the bounce the following expansion holds

$$
f_{\mathrm{B}}(\chi)=\rho_{c}+\frac{1}{3}\left(\frac{2 V_{\mathrm{B}}+3 \alpha}{V_{\mathrm{B}}+3 \alpha}\right) \chi^{2}+\mathcal{O}\left(\chi^{4}\right),
$$

8 It is worth remarking that $\alpha$ and $\beta$ are defined only up to arbitrary constant rescalings of the comoving volume $V_{0}$, which was set equal to one above. We have in general $V=V_{0} a^{3}$. Under the transformation $V_{0} \rightarrow k V_{0}$ with constant $k, \alpha$ and $\beta$ transform according to $\alpha \rightarrow k \alpha, \beta \rightarrow k^{2} \beta$. Thus, the scale invariance property of the standard Friedmann equation is preserved by the quantum corrections. In the GFT formalism such rescaling properties correspond to the invariance of the dynamics under constant rescalings of the number of quanta, cf. Ref. [5,6]. 
where $V_{\mathrm{B}}=-3 \alpha+\sqrt{9 \alpha^{2}+6 \beta}$ is the volume at the bounce and $\rho_{c}=\frac{p_{\psi}^{2}}{2 V_{\mathrm{B}}^{2}}$. For the asymptotic expansion of $f(\chi)$ around the branching points at maximum expansion rate $|\chi|=\chi_{\mathrm{m}}$, see Ref. [22]. Both $f$ and $f_{\chi}$ are continuously matched at the branching points. However, the second derivative $f_{\chi \chi}$ has a discontinuity there: this is a general property of mimetic gravity theories with a limiting curvature scale. Nevertheless, the effective pressure $\tilde{P}(\chi)$ is guaranteed to be finite even when $f_{\chi \chi}$ diverges, since Equation (8) implies

$$
\tilde{P}\left( \pm \chi_{\mathrm{m}}\right)=-(\rho+P)=-(w+1) \rho .
$$

When the universe is large (i.e., in the regime $\chi, \eta \sim 0$ ) one has the expansion (disregarding the linear term, which does not affect the equations of motion)

$$
f_{\mathrm{L}}(\chi)=\sqrt{\frac{2}{3}} \frac{\alpha}{p_{\psi}}|\chi|^{3}-\frac{4}{p_{\psi}^{2}}\left(\alpha^{2}+\frac{1}{9} \beta\right) \chi^{4}+\mathcal{O}\left(|\chi|^{5}\right)
$$

which can be rewritten as

$$
f_{\mathrm{L}}(\chi)=\frac{\alpha}{2 V_{*}} \sqrt{2+\frac{6 \alpha}{V_{*}}} \frac{|\chi|^{3}}{\chi_{\mathrm{m}}}-\frac{\left(V_{*}+3 \alpha\right)\left(V_{*}^{2}+9 \alpha V_{*}+108 \alpha^{2}\right)}{36 V_{*}^{3}} \frac{\chi^{4}}{\chi_{\mathrm{m}}^{2}}+\mathcal{O}\left(|\chi|^{5}\right),
$$

where $\chi_{\mathrm{m}}=\frac{p_{\psi}}{2 V_{*}} \sqrt{3+\frac{9 \alpha}{V_{*}}}$, and $V_{*}=\frac{1}{2}\left(\sqrt{81 \alpha^{2}+48 \beta}-9 \alpha\right)$ is the volume at $\chi=\chi_{\mathrm{m}}$. Please note that if $\alpha=0$, the next non-vanishing term in the expansion is $\mathcal{O}\left(\chi^{6}\right)$.

Once the function $f(\chi)$ has been reconstructed from a given background evolution, one can also consider different matter species coupled to gravity. It must be pointed out that when matter species other than a minimally coupled massless scalar field are considered, parameters such as $p_{\psi}$ and $V_{\mathrm{B}}$ in Equation (32) lose their usual interpretation. This is to be expected, since the relation between $\chi$ and $\eta$ will be different from Equation (30) in the general case. Nevertheless, the values of the critical energy density $\rho_{c}$ and the maximum expansion rate $\chi_{\mathrm{m}}$ are not affected by the different matter species, and represent universal features of the model.

Let us now assume hydrodynamic matter with constant equation of state parameter $w$. Comparing Equations (36) and (25), at late times we obtain a simple description of the effective fluid corresponding to the mimetic gravity corrections as a sum of perfect fluid contributions, each with a constant equation of state. Specifically, we find for the third order term in Equation (36) $\tilde{w}_{3}=\frac{1}{2}(3 w+1)$, whereas for the fourth order term we have $\tilde{w}_{4}=2 w+1$. Clearly, for a massless scalar field $w=1$ one recovers the effective fluid corrections given in Equation (30).

\section{A Special Case: Reproducing the LQC Effective Dynamics}

The case $\alpha=0$ is special and deserves being discussed separately. In fact, in this case one recovers the model of Ref. [21], which reproduces the effective dynamics of LQC for a spatially flat, isotropic universe. After locally inverting $\chi=\chi(\eta)$, one finds the two branches of the function $f(\chi)$

$$
\begin{aligned}
& f_{\mathrm{B}}=\frac{2}{3} \chi_{\mathrm{m}}^{2}\left\{1+\frac{1}{2} q^{2}+\sqrt{1-q^{2}}+q \arcsin (q)\right\}, \\
& f_{\mathrm{L}}=\frac{2}{3} \chi_{\mathrm{m}}^{2}\left\{1+\frac{1}{2} q^{2}-\sqrt{1-q^{2}}-|q|(\arcsin |q|-\pi)\right\},
\end{aligned}
$$

where $\chi_{\mathrm{m}}=p_{\psi} \sqrt{\frac{3}{48 \beta}}$ and we defined $q=\frac{\chi}{\chi_{\mathrm{m}}}$ to make the notation lighter. It must be noted that Equations (37) and (38) do not make any reference to the scalar field $\psi$, which was assumed as the only matter species coupled to gravity in the derivation of Equation (29) in Ref. [5,6]. Thus, for $\alpha=0$ the effective Friedmann equation will take the same universal form regardless of the matter species considered. Using Equation (18), the critical energy density is determined as $\rho_{c}=$ $f_{\mathrm{B}}(0)=\frac{4}{3} \chi_{\mathrm{m}}^{2}$. The energy density of the effective fluid can be computed using Equation (9); the result 
is $\tilde{\rho}=-\frac{\rho_{c}}{2}\left(1-\frac{q^{2}}{2} \pm \sqrt{1-q^{2}}\right)$, where the upper sign corresponds to the bounce branch and the lower one corresponds to a large universe. After some straightforward algebraic manipulations, the Friedmann Equation (7) can then be recast in the following form

$$
\frac{1}{3} \chi^{2}=\rho\left(1-\frac{\rho}{\rho_{c}}\right)
$$

where $\rho$ denotes the total energy density of all matter species that are present. Similarly, using Equations (8) and (10) we can obtain the equation for $\dot{\chi}$. We have, for a general $f(\chi)$

$$
\left(1-\frac{3}{2} f_{\chi \chi}\right) \dot{\chi}=-\frac{3}{2}(\rho+P) .
$$

The bracket to the r.h.s. of Equation (40) can be evaluated using Equations (37) and (38)

$$
1-\frac{3}{2} f_{\chi \chi}=\mp \frac{1}{\sqrt{1-q^{2}}}=\left(1-\frac{2 \rho}{\rho_{c}}\right)^{-1}
$$

where we used Equation (39) in the last equality. Finally, we have

$$
\dot{\chi}=-\frac{3}{2}(\rho+P)\left(1-\frac{2 \rho}{\rho_{c}}\right) .
$$

Thus, the time derivative of the expansion is positive for $\frac{\rho_{c}}{2}<\rho \leq \rho_{c}$ (super-inflation). This is to be contrasted with general relativity, where one always has $\dot{\chi}<0$ for matter satisfying the NEC. Equations (39) and (42) coincide with the effective dynamics of (flat, isotropic) LQC, see e.g., Ref. [4].

It is important to observe that one must change branch of $f(\chi)$ when $\dot{\chi}=0$ [24]. This happens when the density reaches the value $\frac{\rho_{c}}{2}$, see Equation (42), whereby the expansion attains its extremum $\chi^{2}=\chi_{\mathrm{m}}^{2}$. It must be noted that in both branches, as given by Equations (37) and (38), $f_{\chi \chi}$ diverges as $|\chi| \rightarrow \chi$ m; however, the effective pressure $\tilde{P}$ is continuous in the limit since $\tilde{P}=-\frac{\rho}{\rho_{c}}(\rho+2 P)$.

Exact solutions of the effective Friedmann Equation (39) can be derived for hydrodynamic matter (see Ref. [21])

$$
a(t)=a_{\mathrm{B}}\left(1+\frac{3}{4} \rho_{c}(w+1)^{2}\left(t-t_{\mathrm{B}}\right)^{2}\right)^{\frac{1}{3(1+w)}},
$$

where the origin of time has been set to have the bounce at $t=0$. Provided that matter satisfies the NEC, one finds for the bounce duration (defined to have $\chi(T)=\chi_{\mathrm{m}}$ )

$$
T=\frac{1}{\chi_{\mathrm{m}}(1+w)},
$$

which is in good agreement with the estimate given by Equation (23).

Finally, the expansions (33) and (36) for $\alpha=0$ become, respectively

$$
f_{\mathrm{B}}(\chi)=\rho_{c}+\frac{2}{3} \chi^{2}+\mathcal{O}\left(\chi^{4}\right),
$$

and

$$
f_{\mathrm{L}}(\chi)=-\frac{1}{36} \frac{\chi^{4}}{\chi_{\mathrm{m}}^{2}}+\mathcal{O}\left(\chi^{6}\right) .
$$

\section{Anisotropies near the Bounce}

In this Section we generalize the analysis of Ref. [21], studying the evolution of anisotropies near the bounce in a non-singular Bianchi I spacetime, for the model of Section 4 and in the presence of hydrodynamic matter with generic equation of state. 
The line element of Bianchi I in proper time gauge is

$$
\mathrm{d} s^{2}=\mathrm{d} t^{2}-a^{2}(t) \sum_{i} \mathrm{e}^{2 \beta_{(i)}(t)}\left(\mathrm{d} x^{i}\right)^{2},
$$

where $a(t)$ is the mean scale factor, and the variables $\beta_{(i)}$ representing the anisotropies satisfy $\sum_{i} \beta_{(i)}=0$. We will assume hydrodynamical matter with barotropic equation of state. Using the field Equations (3), it can be shown that the $\beta_{(i)}$ evolve according to

$$
\ddot{\beta}_{(i)}+\chi \dot{\beta}_{(i)}=0 .
$$

The solution of Equation (48) gives

$$
\dot{\beta}_{(i)}=\frac{\lambda_{(i)}}{a^{3}(t)},
$$

with $\lambda_{(i)}$ integration constants satisfying $\sum_{i} \lambda_{(i)}=0$. The field equations lead to an effective Friedmann equation for the mean scale factor, which includes the contribution of anisotropies

$$
\frac{1}{3} \chi^{2}=\rho+\tilde{\rho}+\frac{1}{2} \sum_{i} \dot{\beta}_{(i)}^{2} .
$$

The last term of Equation (50) represents the effective energy density of anisotropies (cf. e.g., Ref. [48]), which will be denoted by $\rho_{\Sigma}$. Using Equation (49), we have

$$
\rho_{\Sigma}=\frac{\Sigma^{2}}{2 a^{6}},
$$

having defined the shear scalar as $\Sigma^{2}=\sum_{i} \lambda_{(i)}^{2}$. Thus, the contribution of anisotropies to the modified Friedmann equation is described as a perfect fluid with stiff equation of state $w=1$, as in general relativity.

The evolution of anisotropies, as represented by the $\beta_{(i)}$, is obtained by integrating Equation (49)

$$
\beta_{(i)}(t)=\lambda_{(i)} \int \frac{\mathrm{d} t}{a^{3}(t)},
$$

where $a(t)$ in the integrand is a solution of Equation (50). In the remainder of this Section, we will determine the evolution of anisotropies during the bounce phase for the function $f(\chi)$ given by Equation (32). Since we are only interested in the region around the bounce, it is convenient to use the expansion (33). The energy density of the effective fluid then reads as

$$
\tilde{\rho} \simeq-\rho_{c}+\frac{1}{3}\left(\frac{2 V_{\mathrm{B}}+3 \alpha}{V_{\mathrm{B}}+3 \alpha}\right) \chi^{2} .
$$

The effective Friedmann equations in this regime can then be recast as

$$
\begin{aligned}
\frac{\chi^{2}}{3} & \simeq\left(\frac{V_{\mathrm{B}}+3 \alpha}{V_{\mathrm{B}}}\right)\left(\rho_{c}-\rho-\rho_{\Sigma}\right), \\
\dot{\chi} & \simeq \frac{3}{2}\left(\frac{V_{\mathrm{B}}+3 \alpha}{V_{\mathrm{B}}}\right)\left(\rho+p+2 \rho_{\Sigma}\right) .
\end{aligned}
$$


At the bounce, the scale factor attains its minimum $a_{\mathrm{B}}$, and the r.h.s. of Equation (54) must vanish. We can use this condition to determine the energy density of matter at the bounce $\rho_{B}$ (not to be confused with the critical energy density $\rho_{c}$, which includes the contribution of anisotropies). We have

$$
\rho_{B}=\rho_{\mathcal{c}}-\rho_{\Sigma, \mathrm{B}},
$$

with $\rho_{\Sigma, \mathrm{B}}=\frac{\Sigma^{2}}{2 a_{\mathrm{B}}^{6}}$ being the energy density of anisotropies at the bounce. The r.h.s. of Equation (54) can be expanded around $a_{\mathrm{B}}$; taking into account that $\rho=\rho_{B}\left(\frac{a_{B}}{a}\right)^{3(w+1)}$, this gives

$$
\frac{\chi^{2}}{3} \simeq 3\left(\frac{V_{\mathrm{B}}+3 \alpha}{V_{\mathrm{B}}}\right)\left(\rho_{B}(w+1)+2 \rho_{\Sigma, \mathrm{B}}\right)\left(\frac{a}{a_{\mathrm{B}}}-1\right) .
$$

Taking into account Equation (56), we can rewrite Equation (57) as

$$
\frac{\chi^{2}}{3} \simeq 3(w+1)\left(\frac{V_{\mathrm{B}}+3 \alpha}{V_{\mathrm{B}}}\right)\left(\rho_{c}-\frac{w-1}{w+1} \rho_{\Sigma, \mathrm{B}}\right)\left(\frac{a}{a_{\mathrm{B}}}-1\right) .
$$

The solution is

$$
a(t) \simeq a_{\mathrm{B}}\left(1+\frac{1}{4} \Omega^{2} t^{2}\right)
$$

where we defined

$$
\Omega^{2}=(w+1)\left(\frac{V_{\mathrm{B}}+3 \alpha}{V_{\mathrm{B}}}\right)\left(\rho_{c}-\frac{w-1}{w+1} \rho_{\Sigma, \mathrm{B}}\right) .
$$

The solution (59) for the scale factor shows that regardless of the presence of anisotropies, the model features a first-order bounce, according to the definition given in Ref. [41]. From Equation (59), we find that the mean expansion rate evolves as

$$
\chi(t) \simeq \frac{3}{2} \Omega^{2} t .
$$

Finally, using Equations (59) and (52) we find that the $\beta_{(i)}$ evolve linearly during the bounce

$$
\beta_{(i)}(t) \simeq \beta_{(i)}^{0}+\frac{\lambda_{(i)}}{a_{\mathrm{B}}^{3}} t
$$

where $\beta_{(i)}^{0}$ are integration constants. Our solution (62) shows that anisotropies stay bounded during the bounce, and can be kept under control by means of a suitable choice of parameters for the model. It is interesting to compare this result with a similar one obtained in Ref. [48] for a non-singular bouncing model based on kinetic gravity braiding theories [49].

\section{Effective Gravitational Constant(s)}

The cosmological background equations of mimetic gravity, Equations (7) and (8), can be recast in an alternative form which makes no reference to perfect fluids. The effects introduced by the function $f(\chi)$ in the action (1) are then included in two effective gravitational "constants" $G_{\mathrm{F}}^{\text {eff }}$ and $G_{\mathrm{R}}^{e f f}$, representing respectively the effective coupling of matter to gravity in the Friedmann and the Raychaudhuri equations

$$
\begin{aligned}
& \frac{1}{3} \chi^{2}=8 \pi G_{\mathrm{F}}^{e f f}(\chi) \rho, \\
& \dot{\chi}=-12 \pi G_{\mathrm{R}}^{e f f}(\chi)(\rho+P) .
\end{aligned}
$$


The effective couplings are functions of the expansion rate, and are defined as

$$
\begin{aligned}
& 8 \pi G_{\mathrm{F}}^{e f f}(\chi)=\left(1-3 \frac{\mathrm{d}}{\mathrm{d} \chi}\left(\frac{f}{\chi}\right)\right)^{-1}, \\
& 8 \pi G_{\mathrm{R}}^{e f f}(\chi)=\left(1-\frac{3}{2} f_{\chi \chi}\right)^{-1} .
\end{aligned}
$$

It is worth remarking that variable gravitational constants arise in this framework despite of the fact that the action (1) contains no dilaton couplings. In fact, the reformulation provided here hinges on the presence of a function of the expansion rate $f(\chi)$.

From Equations (63) and (64), and the continuity equation for matter, we find the following equation relating the change of $G_{\mathrm{F}}^{\text {eff }}$ over time to the difference between the two gravitational constants

$$
\dot{G}_{\mathrm{F}}^{e f f} \rho=\chi\left(G_{\mathrm{F}}^{e f f}-G_{\mathrm{R}}^{e f f}\right)(\rho+p) .
$$

We observe that $G_{\mathrm{F}}^{\text {eff }}=G_{\mathrm{R}}^{\text {eff }}$ if and only if $f(\chi)=k_{1} \chi+\frac{k_{2}}{2} \chi^{2}$. In this case, the linear term in $\chi$ has no effect, while the quadratic one leads to a finite redefinition of the Newton constant $8 \pi G_{\mathrm{F}}^{\text {eff }}=\left(1-\frac{3}{2} k_{2}\right)^{-1}$ (see Ref. [37]); thus, in a large universe we must require $k_{2}<\frac{2}{3}$ to ensure that the gravitational interaction remains attractive. ${ }^{9}$ In the general case, both $G_{\mathrm{F}}^{\text {eff }}$ and $G_{\mathrm{R}}^{\text {eff }}$ will evolve with $\chi$. For instance, assuming that in the large universe branch one has $f(\chi) \simeq k \chi^{p}$ with $p>2$ to leading order in $\chi$, leads to

$$
\begin{aligned}
& 8 \pi G_{\mathrm{F}}^{e f f}(\chi) \simeq 1+3 k(p-1) \chi^{p-2}, \\
& 8 \pi G_{\mathrm{R}}^{e f f}(\chi) \simeq 1+\frac{3}{2} k p(p-1) \chi^{p-2} .
\end{aligned}
$$

If we assume that the universe (away from the bounce) is dominated by hydrodynamic matter with equation of state parameter $w$, we have

$$
\begin{aligned}
& 8 \pi G_{\mathrm{F}}^{e f f}(t) \simeq 1+3 k(p-1)\left(\frac{2}{(w+1) t}\right)^{p-2}, \\
& 8 \pi G_{\mathrm{R}}^{e f f}(t) \simeq 1+\frac{3}{2} k p(p-1)\left(\frac{2}{(w+1) t}\right)^{p-2} .
\end{aligned}
$$

The reformulation of the cosmological equations of mimetic gravity offered by Equation (63) and (64) suggests that the coefficients of the leading order terms in the expansion of the branch $f_{\mathrm{L}}$ can be constrained using observational bounds on the time variation of the gravitational constant. We have from Equation (70), for a small $k$ and retaining only the main contribution (corresponding to the radiation dominated era, $w=\frac{1}{3}$ )

$$
\frac{\Delta G_{\mathrm{F}}^{e f f}}{G_{\mathrm{F}}^{e f f}}=1-\frac{G_{\mathrm{F}}^{e f f}\left(t_{\mathrm{BBN}}\right)}{G_{\mathrm{F}}^{e f f}\left(t_{0}\right)} \simeq 3 k(p-1)\left(\frac{3}{2}\right)^{p-2}\left(t_{\mathrm{BBN}}\right)^{2-p} .
$$

where $t_{0}$ is the age of the universe and $t_{\mathrm{BBN}}$ is the time of nucleosynthesis. Bounds on the time variation of the gravitational constant $G_{\mathrm{F}}^{\text {eff }}$ can be derived from primordial nucleosynthesis: $-0.10<\frac{\Delta G_{\mathrm{F}}^{\text {eff }}}{G_{\mathrm{F}}^{\text {eff }}}<0.13[50,51]$. For a given $p>2$, such a bound can be translated into a constraint on $k$. However, such a constraint is very weak for bouncing models. In fact, if the limiting curvature

9 This must be contrasted with the case of bouncing models examined in Sections 3.2 and 4, where the coefficient of the quadratic term must satisfy an opposite inequality in order to guarantee that gravity becomes repulsive at the bounce. 
hypothesis is made, dimensional arguments suggest that $k \sim \chi_{\mathrm{m}}^{2-p}$. This is in fact the case for the models considered in Section 4, see Equations (46) and (36). Moreover, typically one has for the limiting value of the expansion rate $\chi_{\mathrm{m}} \sim t_{\mathrm{Pl}}^{-1}$, where $t_{\mathrm{Pl}}$ is Planck time. Therefore, the time variation of the gravitational constant is extremely small in such models $\frac{\Delta G_{\mathrm{F}}^{\text {eff }}}{G_{\mathrm{F}}^{\text {eff }}} \sim\left(\frac{t_{\mathrm{Pl}}}{t_{\mathrm{BBN}}}\right)^{p-2}$.

A more detailed investigation of the phenomenological consequences of the time variation of $G_{\mathrm{F}}^{\text {eff }}$ and $G_{\mathrm{R}}^{e f f}$ is beyond the scope of the present article and will be left for future work.

\section{Instabilities}

Our presentation of mimetic gravity would not be complete without a discussion of perturbative instabilities. Instabilities of cosmological perturbations for the mimetic gravity theory with action (1) have been studied in Refs. [36,52] for a generic $f(\chi)$; for earlier studies focused on the case of a quadratic $f$ see Ref. $[53,54] .{ }^{10}$ Compared to general relativity, the theory has one extra propagating scalar degree of freedom, whose speed of sound is given by

$$
c_{s}^{2}=\frac{1}{2} \frac{f_{\chi \chi}}{1-\frac{3}{2} f_{\chi \chi}} .
$$

Depending on the sign of the speed of sound, the theory has a ghost instability (for $c_{s}^{2}>0$ ) or a gradient instability (for $c_{s}^{2}<0$ ), see references above. The propagation speed of tensor perturbations is not affected by the term $f(\chi)$ in the action (1). ${ }^{11}$

In the following we will assume that the analytic properties of the function $f(\chi)$ are such as to accommodate for a bouncing background. Some general conclusions can then be drawn on the profile of the speed of sound as a function of the expansion, based on the results derived in Section 3.2. In fact, around the bounce $f(\chi)$ must admit the expansion (19). Moreover, since $\dot{\chi}>0$ in a neighborhood of the bounce, Equation (40) implies that we must have $\vartheta>\frac{2}{3}$, provided that ordinary matter fields satisfy the NEC. Thus, at the bounce we have

$$
c_{s}^{2}=\frac{\vartheta}{2-3 \vartheta}<0,
$$

which corresponds to a gradient instability. The expansion rate attains its extremum at $|\chi|=\chi_{\mathrm{m}}$, where two different branches of the multivalued function $f(\chi)$ are joined together; at that point the second derivative $f_{\chi \chi}$ is divergent, whereby the speed of sound squared takes the universal value $c_{s}^{2}=-\frac{1}{3}$. We conclude that a generic feature of bouncing models in mimetic gravity is that the bounce is always accompanied by a gradient instability of scalar perturbations, which extends beyond the onset of the standard decelerated expansion. The possibility that $c_{s}^{2}$ may turn to positive values at a later stage is not excluded, but depends on the details of the model, and specifically on the functional form of the branch $f_{\mathrm{L}}(\chi)$ corresponding to a large universe.

It is interesting to study the behavior of $c_{s}^{2}$ in the models examined in Section 4 , where a bouncing background is explicitly realized. To begin with, let us start from the special case $\alpha=0$, which reproduces the LQC effective dynamics for the cosmological background. The two branches $f_{\mathrm{B}}, f_{\mathrm{L}}$ in this case are given by Equations (37) and (38), respectively. We find, using Equations (73) and (41)

$$
c_{s}^{2}=-\frac{1}{3}\left(1 \pm \sqrt{1-q^{2}}\right)=-\frac{2}{3} \frac{\rho}{\rho_{c}} .
$$

10 It must be noted that the quadratic case is equivalent with the IR limit of projectable Hořava-Lifshitz gravity [54], see also Ref. [55].

11 The situation is different in other versions of mimetic gravity, see e.g., [23] for a general analysis based on the DHOST formulation of (extended) mimetic gravity theories. 
In the second step of (75), the upper sign corresponds to $f_{\mathrm{B}}$, whereas the lower one corresponds to $f_{\mathrm{L}}$. We note that the speed of sound squared is always negative, has a minimum at the bounce $\left(c_{s}^{2}\right)_{\min }=-\frac{2}{3}$ when $\rho=\rho_{c}$, and approaches zero from below as $\rho \rightarrow 0$. Given Equation (75), and recalling that maximal expansion rate in this model is reached at $\rho=\frac{\rho_{c}}{2}$, it is straightforward to check the general feature $c_{S}^{2}\left( \pm \chi_{\mathrm{m}}\right)=-\frac{1}{3}$. We observe that $c_{S}^{2}$ is negative throughout cosmic history for the model with $\alpha=0$, and approaches zero from below in the large universe branch as $\chi$ tends to zero (cf. Ref. [25]). It is interesting to compare these results with those obtained in Ref. [56] for a model based on generalized Galileons [57], where the speed of sound squared becomes negative-although only for a short period-around the bounce; see also Ref. [58,59] for a comparison between such effective models and the dynamics of perturbations in LQC. In the models cited above gradient instabilities arise due to the violation of the NEC at the bounce (see also [60] and references therein). Recently, the possibility of establishing a theoretical no-go theorem regarding the realization of a healthy non-singular bounce (i.e., free of pathologies such as gradient instabilities) has been discussed in the context of generalized Galileons, see Refs. [61-63].

The example examined above is just a particular case of the model reproducing the background dynamics of GFT condensates, studied in Section 4 . In the general case, i.e., for $\alpha \neq 0$, we have at the bounce

$$
\left(c_{s}^{2}\right)_{\min }=-\frac{2}{3}\left(1+\frac{\alpha}{V_{\mathrm{B}}}\right) .
$$

In the large universe branch instead and for $\chi \simeq 0$ we have, to leading order in $\chi$

$$
c_{S}^{2} \simeq \frac{3 \alpha}{V_{*}} \sqrt{2+\frac{6 \alpha}{V_{*}}} \frac{|\chi|}{\chi_{\mathrm{m}}} .
$$

Thus, $c_{S}^{2}$ and $\alpha$ have the same sign in this regime. Therefore, for $\alpha<0$ the situation is qualitatively similar to the $\alpha=0$ case examined above, with a gradient instability extending also to the large universe branch. For $\alpha>0$ the situation is different: there is a cross-over from $c_{s}^{2}<0$ near the bounce to $c_{S}^{2}>0$ when the universe is large. Such a cross-over must necessarily take place after the universe enters the phase of decelerated expansion, since $c_{s}^{2}=-\frac{1}{3}$ when $\dot{\chi}=0$ (see above). Thus, while the bounce is always accompanied by a gradient instability, the late universe branch would be characterized by a ghost instability for $\alpha>0$. We remark that the cross-over point where $c_{S}^{2}=0$ corresponds to a regime of strong coupling [54].

\section{Discussion}

We conclude by reviewing the main results obtained in this work and indicating directions for future studies.

In Section 3 we illustrated in complete generality the theory reconstruction procedure for the function $f(\chi)$ in mimetic gravity. In the case of bouncing backgrounds, the implementation of the limiting curvature hypothesis requires that $f(\chi)$ be multivalued. This case was carefully examined and we gave general prescriptions to ensure continuity of $f(\chi)$ and its first derivative along the cosmic trajectory; in particular, by imposing suitable matching conditions at the branching points, both the energy density $\tilde{\rho}$ and pressure $\tilde{P}$ of the effective fluid are continuous throughout cosmic history. We showed that local properties of the function $f(\chi)$ are directly related to physically relevant quantities characterizing the evolution of the cosmic background, such as the critical energy density and the bounce duration, as well as the equation of state of the effective fluid. In particular, the latter was shown to approach a constant value at late times, which is determined by the dominant matter species and the leading order term in the asymptotic expansion of $f(\chi)$ in that regime.

In Section 4 we focused on a specific model obtained in Ref. [22], where the function $f(\chi)$ was suitably reconstructed in order to reproduce the background evolution obtained from GFT condensates in Ref. [5,6]. Quantities of physical interest were derived from local analysis of the two branches $f_{\mathrm{B}}, f_{\mathrm{L}}$, using the results of Section 3. The special case corresponding to the effective dynamics of LQC for a flat, 
isotropic universe was studied in detail. As an application, we studied the evolution of anisotropies near the bounce in a Bianchi I universe for the model of Ref. [22]: our results generalize those obtained in Ref. [21] and show that anisotropies do not grow significantly during the bounce, and therefore do not spoil the smoothness of the bounce. It would be interesting to compare the results obtained in the effective approach considered here, with those of Ref. [64], where the dynamics of GFT condensates of anisotropic quanta was studied (see also Ref. [65]). As discussed in Ref. [11,29], the evolution of anisotropies is qualitatively similar in LQC and the corresponding mimetic gravity theory. It is, therefore, natural to ask whether an analogous statement can be made for GFT cosmology and the related model in mimetic gravity. We leave this question for future work. Spherically symmetric geometries are also of interest and can be studied in the present framework by extending the analysis of Refs. $[27,28]$.

In Section 6 we showed that there is an interesting reformulation of mimetic gravity involving two distinct time-varying effective gravitational constants $G_{\mathrm{F}}^{e f f}$ and $G_{\mathrm{R}}^{\text {eff }}$, featuring respectively in the Friedmann and the Raychaudhuri equations. Consistency of such a description with the Bianchi identities is ensured by Equation (67), which is identically satisfied in mimetic gravity by all choices of the function $f(\chi)$. We derived the time evolution of the effective gravitational constants during the phase of decelerated expansion for $f(\chi) \sim \chi^{p}$, with $p>2$. We showed that the predicted time variation is too small to be observed if the limiting curvature hypothesis is realized. It would be of interest to further explore the consequences of the time variation of $G_{\mathrm{F}}^{e f f}$ and $G_{\mathrm{R}}^{e f f}$ in a more general and model independent setting.

Our discussion of perturbative instabilities in Section 7 highlights some serious limitations of bouncing models in mimetic gravity, which may hinder the possibility of using the simplest framework with the covariant action (1) for an effective description of quantum gravity in inhomogeneous spacetimes. The presence of gradient or ghost instabilities, which is a distinctive feature of mimetic gravity, seems to be even more serious in bouncing cosmologies; in fact, in such models the infinite age of the universe would offer no chance to keep instabilities under control. Remarkably, this issue has not been much appreciated in the literature on bouncing cosmologies in mimetic gravity. Based on the analogy with LQC (see Ref. [58]), we expect the bounce to be accompanied by a short-lived gradient instability around the bounce affecting short-wavelength modes; however, there should be no instabilities away from the bounce. Some proposals to cure the instabilities by means of further modification of the mimetic gravity action have been made in Refs. [66,67]; however, their correspondence with the effective dynamics of quantum gravity models is yet to be established and shall be investigated in future work.

Funding: This work was partially supported by the Atlantic Association for Research in the Mathematical Sciences (AARMS) and by the Natural Sciences and Engineering Research Council of Canada (NSERC).

Acknowledgments: It is a pleasure to thank Sabir Ramazanov and Edward Wilson-Ewing for helpful discussions on instabilities in mimetic gravity.

Conflicts of Interest: The authors declare no conflict of interest.

\section{References}

1. Brandenberger, R.; Peter, P. Bouncing Cosmologies: Progress and Problems. Found. Phys. 2017, 47, 797-850. [CrossRef]

2. Cai, Y.F. Exploring Bouncing Cosmologies with Cosmological Surveys. Sci. China Phys. Mech. Astron. 2014, 57, 1414-1430. [CrossRef]

3. Bojowald, M. Loop quantum cosmology. Living Rev. Rel. 2008, 11, 4. [CrossRef]

4. Ashtekar, A.; Singh, P. Loop Quantum Cosmology: A Status Report. Class. Quant. Grav. 2011, $28,213001$. [CrossRef]

5. Oriti, D.; Sindoni, L.; Wilson-Ewing, E. Emergent Friedmann dynamics with a quantum bounce from quantum gravity condensates. Class. Quant. Grav. 2016, 33, 224001. [CrossRef] 
6. Oriti, D.; Sindoni, L.; Wilson-Ewing, E. Bouncing cosmologies from quantum gravity condensates. Class. Quant. Grav. 2017, 34, 04LT01. [CrossRef]

7. Alesci, E.; Botta, G.; Cianfrani, F.; Liberati, S. Cosmological singularity resolution from quantum gravity: The emergent-bouncing universe. Phys. Rev. D 2017, 96, 046008. [CrossRef]

8. Gielen, S.; Oriti, D. Cosmological perturbations from full quantum gravity. Phys. Rev. D 2018, 98, 106019. [CrossRef]

9. Gerhardt, F.; Oriti, D.; Wilson-Ewing, E. Separate universe framework in group field theory condensate cosmology. Phys. Rev. D 2018, 98, 066011. [CrossRef]

10. Gielen, S. Inhomogeneous universe from group field theory condensate. J. Cosmol. Astropart. Phys. 2019, 2019, 013. [CrossRef]

11. Bodendorfer, N.; Schäfer, A.; Schliemann, J. Canonical structure of general relativity with a limiting curvature and its relation to loop quantum gravity. Phys. Rev. D 2018, 97, 084057. [CrossRef]

12. Langlois, D.; Liu, H.; Noui, K.; Wilson-Ewing, E. Effective loop quantum cosmology as a higher-derivative scalar-tensor theory. Class. Quant. Grav. 2017, 34, 225004. [CrossRef]

13. Markov, M. Limiting density of matter as a universal law of nature. J. Exp. Theor. Phys. Lett. 1982, 36, 265.

14. Markov, M. Possible state of matter just before the collapse stage. J. Exp. Theor. Phys. Lett. 1987, 46, 431.

15. Frolov, V.P.; Markov, M.A.; Mukhanov, V.F. Black Holes as Possible Sources of Closed and Semiclosed Worlds. Phys. Rev. D 1990, 41, 383. [CrossRef]

16. Frolov, V.P.; Markov, M.A.; Mukhanov, V.F. Through a black hole into a new universe? Phys. Lett. B 1989, 216, 272-276. [CrossRef]

17. Mukhanov, V.F.; Brandenberger, R.H. A Nonsingular universe. Phys. Rev. Lett. 1992, 68, 1969-1972. [CrossRef] [PubMed]

18. Brandenberger, R.H.; Mukhanov, V.F.; Sornborger, A. A Cosmological theory without singularities. Phys. Rev. D 1993, 48, 1629-1642. [CrossRef]

19. Easson, D.A.; Brandenberger, R.H. Nonsingular dilaton cosmology in the string frame. J. High Energy Phys. 1999, 1999, 003. [CrossRef]

20. Yoshida, D.; Quintin, J.; Yamaguchi, M.; Brandenberger, R.H. Cosmological perturbations and stability of nonsingular cosmologies with limiting curvature. Phys. Rev. D 2017, 96, 043502. [CrossRef]

21. Chamseddine, A.H.; Mukhanov, V. Resolving Cosmological Singularities. J. Cosmol. Astropart. Phys. 2017, 2017, 009. [CrossRef]

22. de Cesare, M. Limiting curvature mimetic gravity for group field theory condensates. Phys. Rev. D 2019, 99, 063505. [CrossRef]

23. Langlois, D.; Mancarella, M.; Noui, K.; Vernizzi, F. Mimetic gravity as DHOST theories. J. Cosmol. Astropart. Phys. 2019, 2019, 036. [CrossRef]

24. Brahma, S.; Golovnev, A.; Yeom, D.H. On singularity-resolution in mimetic gravity. Phys. Lett. B 2018, 782, 280-284. [CrossRef]

25. De Haro, J.; Aresté Saló, L.; Pan, S. Limiting curvature mimetic gravity and its relation to Loop Quantum Cosmology. arXiv 2018, arXiv:gr-qc/1803.09653.

26. De Haro, J.; Pan, S. Note on bouncing backgrounds. Phys. Rev. D 2018, 97, 103518. [CrossRef]

27. Chamseddine, A.H.; Mukhanov, V. Nonsingular Black Hole. Eur. Phys. J. C 2017, 77, 183. [CrossRef]

28. Ben Achour, J.; Lamy, F.; Liu, H.; Noui, K. Non-singular black holes and the Limiting Curvature Mechanism: A Hamiltonian perspective. J. Cosmol. Astropart. Phys. 2018, 2018, 072. [CrossRef]

29. Bodendorfer, N.; Mele, F.M.; Münch, J. Is limiting curvature mimetic gravity an effective polymer quantum gravity? Class. Quant. Grav. 2018, 35, 225001. [CrossRef]

30. Chamseddine, A.H.; Mukhanov, V. Mimetic Dark Matter. J. High Energy Phys. 2013, 2013, 135. [CrossRef]

31. Deruelle, N.; Rua, J. Disformal Transformations, Veiled General Relativity and Mimetic Gravity. J. Cosmol. Astropart. Phys. 2014, 2014, 002. [CrossRef]

32. Golovnev, A. On the recently proposed Mimetic Dark Matter. Phys. Lett. B 2014, 728, 39-40. [CrossRef]

33. Chamseddine, A.H.; Mukhanov, V.; Vikman, A. Cosmology with Mimetic Matter. J. Cosmol. Astropart. Phys. 2014, 2014, 017. [CrossRef]

34. Sebastiani, L.; Vagnozzi, S.; Myrzakulov, R. Mimetic gravity: A review of recent developments and applications to cosmology and astrophysics. Adv. High Energy Phys. 2017, 2017, 3156915. [CrossRef] 
35. Langlois, D. Dark Energy and Modified Gravity in Degenerate Higher-Order Scalar-Tensor (DHOST) theories: A review. arXiv 2018, arXiv:gr-qc/1811.06271.

36. Firouzjahi, H.; Gorji, M.A.; Hosseini Mansoori, S.A. Instabilities in Mimetic Matter Perturbations. J. Cosmol. Astropart. Phys. 2017, 2017, 031. [CrossRef]

37. Mirzagholi, L.; Vikman, A. Imperfect Dark Matter. J. Cosmol. Astropart. Phys. 2015, 2015, 028. [CrossRef]

38. Brown, J.D.; Kuchar, K.V. Dust as a standard of space and time in canonical quantum gravity. Phys. Rev. D 1995, 51, 5600-5629. [CrossRef]

39. Husain, V.; Pawlowski, T. Time and a physical Hamiltonian for quantum gravity. Phys. Rev. Lett. 2012, 108, 141301. [CrossRef]

40. Husain, V.; Pawlowski, T. Dust reference frame in quantum cosmology. Class. Quant. Grav. 2011, $28,225014$. [CrossRef]

41. Cattoen, C.; Visser, M. Necessary and sufficient conditions for big bangs, bounces, crunches, rips, sudden singularities, and extremality events. Class. Quant. Grav. 2005, 22, 4913-4930. [CrossRef]

42. de Cesare, M.; Pithis, A.G.A.; Sakellariadou, M. Cosmological implications of interacting Group Field Theory models: cyclic Universe and accelerated expansion. Phys. Rev. D 2016, 94, 064051. [CrossRef]

43. Lin, C.; Brandenberger, R.H.; Perreault Levasseur, L. A Matter Bounce By Means of Ghost Condensation. J. Cosmol. Astropart. Phys. 2011, 2011, 019. [CrossRef]

44. Gielen, S. Group field theory and its cosmology in a matter reference frame. Universe 2018, 4, 103. [CrossRef]

45. Adjei, E.; Gielen, S.; Wieland, W. Cosmological evolution as squeezing: A toy model for group field cosmology. Class. Quant. Grav. 2018, 35, 105016. [CrossRef]

46. Wilson-Ewing, E. A relational Hamiltonian for group field theory. arXiv 2018, arXiv:gr-qc/1810.01259.

47. de Cesare, M.; Sakellariadou, M. Accelerated expansion of the Universe without an inflaton and resolution of the initial singularity from Group Field Theory condensates. Phys. Lett. B 2017, 764, 49-53. [CrossRef]

48. Cai, Y.F.; Brandenberger, R.; Peter, P. Anisotropy in a Nonsingular Bounce. Class. Quant. Grav. 2013, 30, 075019. [CrossRef]

49. Deffayet, C.; Pujolas, O.; Sawicki, I.; Vikman, A. Imperfect Dark Energy from Kinetic Gravity Braiding. J. Cosmol. Astropart. Phys. 2010, 2010, 026. [CrossRef]

50. Uzan, J.P. Varying Constants, Gravitation and Cosmology. Living Rev. Rel. 2011, 14, 2. [CrossRef]

51. Cyburt, R.H.; Fields, B.D.; Olive, K.A.; Skillman, E. New BBN limits on physics beyond the standard model from ${ }^{4}$ He. Astropart. Phys. 2005, 23, 313-323. [CrossRef]

52. Takahashi, K.; Kobayashi, T. Extended mimetic gravity: Hamiltonian analysis and gradient instabilities. J. Cosmol. Astropart. Phys. 2017, 2017, 038. [CrossRef]

53. Ijjas, A.; Ripley, J.; Steinhardt, P.J. NEC violation in mimetic cosmology revisited. Phys. Lett. B 2016, 760, 132-138. [CrossRef]

54. Ramazanov, S.; Arroja, F.; Celoria, M.; Matarrese, S.; Pilo, L. Living with ghosts in Hoava-Lifshitz gravity. J. High Energy Phys. 2016, 2016, 020. [CrossRef]

55. Capela, F.; Ramazanov, S. Modified Dust and the Small Scale Crisis in CDM. J. Cosmol. Astropart. Phys. 2015, 2015, 051. [CrossRef]

56. Cai, Y.F.; Easson, D.A.; Brandenberger, R. Towards a Nonsingular Bouncing Cosmology. J. Cosmol. Astropart. Phys. 2012, 2012, 020. [CrossRef]

57. Deffayet, C.; Gao, X.; Steer, D.A.; Zahariade, G. From k-essence to generalised Galileons. Phys. Rev. D 2011, 84, 064039. [CrossRef]

58. Cai, Y.F.; Wilson-Ewing, E. Non-singular bounce scenarios in loop quantum cosmology and the effective field description. J. Cosmol. Astropart. Phys. 2014, 2014, 026. [CrossRef]

59. Cai, Y.F.; Marciano, A.; Wang, D.G.; Wilson-Ewing, E. Bouncing cosmologies with dark matter and dark energy. Universe 2016, 3, 1. [CrossRef]

60. Libanov, M.; Mironov, S.; Rubakov, V. Generalized Galileons: Instabilities of bouncing and Genesis cosmologies and modified Genesis. J. Cosmol. Astropart. Phys. 2016, 2016, 037. [CrossRef]

61. Kobayashi, T. Generic instabilities of nonsingular cosmologies in Horndeski theory: A no-go theorem. Phys. Rev. D 2016, 94, 043511. [CrossRef]

62. Akama, S.; Kobayashi, T. Generalized multi-Galileons, covariantized new terms, and the no-go theorem for nonsingular cosmologies. Phys. Rev. D 2017, 95, 064011. [CrossRef] 
63. Banerjee, S.; Cai, Y.F.; Saridakis, E.N. Evading the theoretical no-go theorem for nonsingular bounces in Horndeski/Galileon cosmology. arXiv 2018, arXiv:gr-qc/1808.01170.

64. de Cesare, M.; Oriti, D.; Pithis, A.G.A.; Sakellariadou, M. Dynamics of anisotropies close to a cosmological bounce in quantum gravity. Class. Quant. Grav. 2018, 35, 015014. [CrossRef]

65. Pithis, A.G.A.; Sakellariadou, M. Relational evolution of effectively interacting group field theory quantum gravity condensates. Phys. Rev. D 2017, 95, 064004. [CrossRef]

66. Gorji, M.A.; Hosseini Mansoori, S.A.; Firouzjahi, H. Higher Derivative Mimetic Gravity. J. Cosmol. Astropart. Phys. 2018, 2018, 020. [CrossRef]

67. Hirano, S.; Nishi, S.; Kobayashi, T. Healthy imperfect dark matter from effective theory of mimetic cosmological perturbations. J. Cosmol. Astropart. Phys. 2017, 2017, 009. [CrossRef]

(C) 2019 by the author. Licensee MDPI, Basel, Switzerland. This article is an open access article distributed under the terms and conditions of the Creative Commons Attribution (CC BY) license (http:// creativecommons.org/licenses/by/4.0/). 